\title{
Quasiperiodically Driven Ratchets for Cold Atoms
}

\author{
R. Gommers, ${ }^{1}$ S. Denisov, ${ }^{2}$ and F. Renzoni ${ }^{1}$ \\ ${ }^{1}$ Department of Physics and Astronomy, University College London, Gower Street, London WC1E 6BT, United Kingdom \\ ${ }^{2}$ Max-Planck-Institut für Physik komplexer Systeme, Nöthnitzer Strasse 38, 01187 Dresden, Germany
}

(Received 26 April 2006; published 22 June 2006)

\begin{abstract}
We investigate experimentally the route to quasiperiodicity in a driven ratchet for cold atoms and examine the relationship between symmetries and transport while approaching the quasiperiodic limit. Depending on the specific form of driving, quasiperiodicity results in the complete suppression of transport, or in the restoration of the symmetries which hold for a periodic driving.
\end{abstract}

PACS numbers: 05.40.Fb, 05.60.Cd, 32.80.Pj

The ratchet effect [1-3], i.e., the possibility of obtaining directed transport of particles in the absence of a net bias force, has recently been attracting considerable interest [48]. Initially introduced to point out the strict limitations on directed transport at equilibrium imposed by the second principle of thermodynamics [9], the ratchet effect has subsequently received much attention as it was identified as a model elucidating the working principle of molecular motors [7]. More recently, considerable activity on ratchets by the condensed matter community was stimulated by the possibility of using the ratchet phenomenon to realize new types of electron pumps [10].

In order to obtain directed transport in the absence of a net bias, the ensemble of particles has to be driven out of equilibrium, so to overcome the restrictions imposed by the second principle of thermodynamics. Additionally, relevant symmetries of the system have to be broken to allow directed transport. Theoretical work [5,6] precisely identified the relationship between symmetries and transport in the case of periodically driven ratchets, and experiments with cold atoms in optical lattices validated the theoretical predictions $[11,12]$. The theoretical analysis was then extended to explore the relationship between symmetries and transport for quasiperiodically driven ratchets, and the general symmetries which forbid directed transport were identified [13,14].

In the present work we investigate experimentally the route to quasiperiodicity in a driven ratchet for cold atoms, and we examine the relationship between symmetries and transport while approaching the quasiperiodic limit. It will be shown that, depending on the specific form of driving, quasiperiodicity may result in the complete suppression of transport, or in the restoration of the symmetries which hold for a periodic driving.

Our experiments are based on caesium atoms cooled and trapped in a near-resonant driven optical lattice [15]. The lattice beam geometry is the same as the one used in our previous experiments [12]: one beam (beam 1) propagates in the $z$ direction; the three other beams (beams 2-4) propagate in the opposite direction, arranged along the edges of a triangular pyramid having the $z$ direction as axis. We refer to Ref. [12] for further details of the setup, and we summarize here only the essential features. The interference between the lattice fields creates a periodic and spatially symmetric potential for the atoms. The interaction with the light also leads to damping of the atomic motion, and the level of dissipation can be varied by changing the lattice parameters (intensity and detuning from atomic resonance). An arbitrary, time-dependent force can be applied onto the atoms by appropriately phase modulating the lattice beams. More precisely, a phase modulation $\alpha(t)$ of lattice beam 1 will result in an inertial force in the reference frame of the optical lattice of the form $F(t)=-m \ddot{\alpha} / k_{z}$, where $k_{z}=2 \pi / \lambda_{z}$ and $\lambda_{z} / 2$ is the distance between neighboring minima in the $z$ direction.

In order to study the relationship between symmetries and transport in the quasiperiodic limit, we consider a multifrequency driving, obtained by combining signals at three different frequencies: $\omega_{1}, 2 \omega_{1}$, and $\omega_{2}$. For $\omega_{2} / \omega_{1}$ irrational, the driving is quasiperiodic. Clearly, in a real experiment $\omega_{2} / \omega_{1}$ is always a rational number, which can be written as $\omega_{2} / \omega_{1}=p / q$, with $p, q$ two coprime positive integers. However, as the duration of the experiment is finite, by choosing $p$ and $q$ sufficiently large it is possible to obtain a driving which is effectively quasiperiodic on the time scale of the experiment. In the present investigation, we consider two different types of driving forces. We analyze the two cases separately, as their analysis requires different symmetry considerations.

In the first examined case, we considered a driving of the following form:

$$
F(t)=A \cos \left(\omega_{1} t\right)+B \cos \left(2 \omega_{1} t+\phi\right)+C \cos \left(\omega_{2} t+\delta\right) .
$$

Such a force can be applied by phase modulating one of the lattice beams in an appropriate way. In the experiment we modulate directly the derivative of the phase, i.e., we apply a frequency modulation of the form

$$
\dot{\alpha}(t)=a \sin \left(\omega_{1} t\right)+b \sin \left(2 \omega_{1} t+\phi\right)+c \sin \left(\omega_{2} t+\delta\right),
$$

which corresponds to a force of the form of Eq. (1) with $A=-m a \omega_{1} / k_{z}, B=-2 m b \omega_{1} / k_{z}$, and $C=-m c \omega_{2} / k_{z}$. 
We consider first the case of truly periodic driving, i.e., a driving which is periodic on the time scale of our experiment. For the current choice of the experimental parameters the atomic motion is weakly damped. We can therefore use the symmetry arguments valid in the dissipationless limit, and then include the corrections due to the damping. For a spatially symmetric potential, and a driving $F(t)$ of period $T$ there are two symmetries which need to be considered to understand the atomic transport. If $F(t)$ is shift symmetric, i.e., $F(t)=-F(t+T / 2)$, the symmetry $S_{a}:(x, t) \rightarrow(-x, t+T / 2)$ is realized. Furthermore, if $F$ is symmetric, i.e., $F(t)=F(-t)$, the symmetry $S_{b}:(x, t) \rightarrow$ $(x,-t)$ is realized. Whenever $S_{a}$ and/or $S_{b}$ are realized, directed transport is forbidden.

As a starting point, we examine the case of biharmonic driving at frequencies $\omega_{1}, 2 \omega_{1}[C=0$ in Eq. (1)]. The driving breaks the shift symmetry for any value of $\phi$, and is time symmetric for $\phi=n \pi$, with $n$ integer. It follows that directed motion is forbidden for $\phi=n \pi$, and indeed previous work $[5,11]$ showed that the current $I$ is of the form $I=I_{0} \sin \phi$, with $I_{0}$ a constant. Now we include the effect of dissipation. Damping breaks the time-reversal symmetry and results in an additional phase shift $\phi_{0}$ of the current as a function of the phase $\phi[5,12]$. The current is in this case $I=I_{0} \sin \left(\phi+\phi_{0}\right)$. Our experimental results for the atomic current as a function of the phase $\phi$ are shown in Fig. 1. The squares represent the case discussed above of a biharmonic drive. These data show the expected dependence of the current on the phase $\phi$ and will serve for reference for the rest of the investigation.

We now introduce a third driving at frequency $\omega_{2}=$ $(p / q) \omega_{1}$ and phase $\delta$; see Eq. (1). In the case of a periodic driving, the same symmetry considerations used above for the biharmonic drive apply. For $\delta=0$ the driving is invariant under time reversal, and this forbids directed transport for $\phi=n \pi$. Instead, for $\delta \neq 0$ the symmetry under time reversal is broken and transport is allowed also for $\phi=n \pi$. In other words, for $\delta \neq 0$ the third driving leads to an additional shift of the current as a function of $\phi$. The magnitude of such a shift depends obviously on the phase $\delta$, which controls the time symmetry of the Hamiltonian at $\phi=n \pi$. To verify this behavior, valid for a periodic driving, we considered the simple situation with the frequency $\omega_{2}$ of the third driving equal to $\omega_{1}\left(\omega_{2}=\omega_{1}\right.$, i.e., $p=q=1$ ) and study the current as a function of $\phi$, for different values of $\delta$. The experimental data, shown in Fig. 1, confirm the behavior predicted on the basis of symmetry considerations. For $\delta=0$ the third driving does not introduce any additional phase shift with respect to the case of biharmonic drive. Instead, the third driving with $\delta \neq 0$ leads to an additional phase shift of the current as a function of $\phi$, and such a phase shift is an increasing function of $\delta$.

We consider now the quasiperiodic limit, which corresponds to an irrational ratio of frequencies $\omega_{2} / \omega_{1}$. Theoretical work showed that in order to analyze the

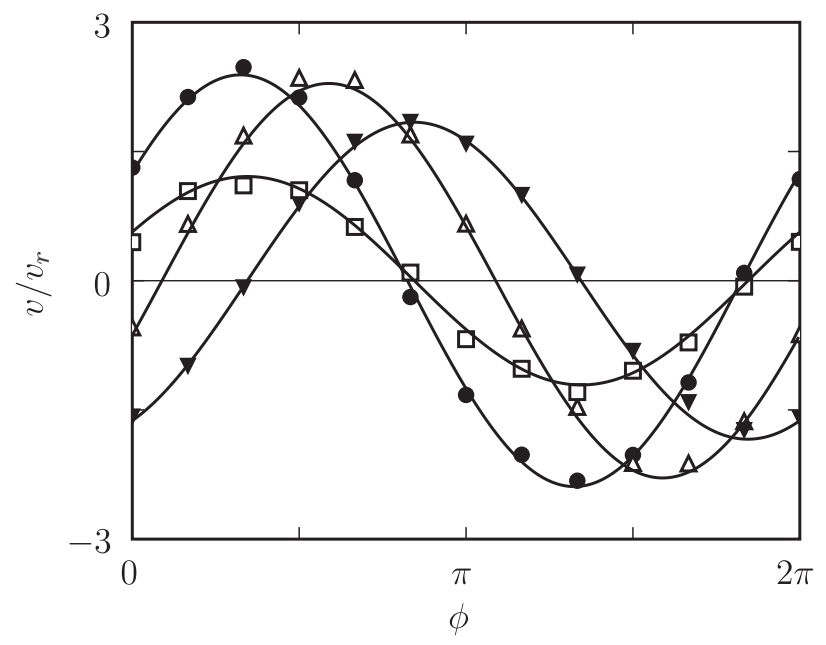

FIG. 1. Experimental results for the average current of atoms in an optical lattice driven by a force of the form of Eq. (1). The average atomic velocity $v$, rescaled by the recoil velocity $v_{r}$, is plotted as a function of the phase $\phi$. The parameters of the optical lattice are the following: the vibrational frequency at the bottom of the well is $\omega_{v}=2 \pi 170 \mathrm{kHz}$; the laser detuning $\Delta=$ $-24 \Gamma$, where $\Gamma$ is the excited state linewidth. The parameters of the driving common to all data sets are $\omega_{1}=\omega_{2}=2 \pi \times$ $100 \mathrm{kHz}, a=b=75 \mathrm{kHz}$. The data set with squares corresponds to a simple biharmonic driving at frequencies $\omega_{1}, 2 \omega_{1}$, i.e., a driving of the form of Eq. (1) with $C=0$. The other data sets correspond to a driving including all three harmonics, with $c=75 \mathrm{kHz}$, and differ for the value of the phase $\delta$. Circles corresponds to $\delta=0$, open triangles to $\delta=\pi / 4$ and solid triangles to $\delta=\pi / 2$. The lines represent the best fit of the data with the function $v=v_{\max } \sin \left(\phi+\phi_{0}\right)$.

relationship between symmetry and transport in the quasiperiodic case, the two phases $\psi_{1} \equiv \omega_{1} t$ and $\psi_{2} \equiv \omega_{2} t$ can be treated as independent variables. The symmetries valid in the periodic case can then be generalized to the quasiperiodic case. The driving force $F(t)$ is said to be shift symmetric if it changes sign under one of the three transformations $\psi_{\alpha} \rightarrow \psi_{\alpha}+\pi$, where $\alpha$ is any subset of $\{1,2\}$, i.e., the $\pi$ shift is applied to either any of the two variables or to both of them. If $F$ is shift symmetric, then the system is invariant under the transformation

$$
\tilde{S}_{a}: x \rightarrow-x, \quad \psi_{\alpha} \rightarrow \psi_{\alpha}+\pi,
$$

and directed motion is forbidden [13,14]. The symmetry for time reversal is generalized in the same way. The driving is said to be symmetric if $F\left(-\psi_{1}+\chi_{1},-\psi_{2}+\right.$ $\left.\chi_{2}\right)=F\left(\psi_{1}, \psi_{2}\right)$, with $\chi_{1}, \chi_{2}$ appropriately chosen constants. If the driving is symmetric, in the dissipationless limit the system is invariant under the transformation

$$
\tilde{S}_{b}: x \rightarrow x, \quad \psi_{j} \rightarrow-\psi_{j}+\chi_{j} \quad(j=1,2),
$$

and directed transport is forbidden [14]. These two symmetries determine the general transport properties in the quasiperiodic limit. For our driving of the form of Eq. (1), 
the shift symmetry is broken for any choice of $\phi$ and $\delta$. The transport is then controlled by the time-reversal symmetry $\tilde{S}_{b}$ [Eq. (4)]. We notice that the driving is invariant under the transformation $\psi_{2} \rightarrow-\psi_{2}+\chi_{2}$ for any $\delta$, as $\delta$ can be reabsorbed in $\chi_{2}$. Therefore the invariance under the transformation $\tilde{S}_{b}$ is entirely determined by the invariance of $F$ under the transformation $\psi_{1} \rightarrow-\psi_{1}+\chi_{1}$; i.e., we recover the result for biharmonic driving: $\tilde{S}_{b}$ is a symmetry, and therefore directed motion is forbidden, for $\phi=n \pi$. In other words, in the quasiperiodic limit the additional driving $C \cos \left(\omega_{2} t+\delta\right)$ does not change the symmetries corresponding to a pure biharmonic driving, independently of the choice of $\delta$.

In order to explore experimentally the route to quasiperiodicity and to investigate the quasiperiodic limit, we studied the atomic current as a function of $\phi$ for $\omega_{2} / \omega_{1}=$ $p / q$, with $p$ and $q$ coprimes. As already discussed, by increasing $p$ and $q$, the driving will be more and more quasiperiodic on the finite duration of the experiment. Correspondingly, we will take $p q$ to characterize the degree of quasiperiodicity. For given $p$ and $q$, we measured the average atomic velocity as a function of $\phi$. By fitting the data with $v=v_{\max } \sin \left(\phi+\phi_{0}\right)$, we determined the phase shift $\phi_{0}$, with results as in Fig. 2. For small values of the product $p q$, i.e., for periodic driving, the third driving at frequency $\omega_{2}$ leads to a shift $\phi_{0}$ which strongly depends on the actual value of $p q$. For larger values of $p q$, i.e., approaching quasiperiodicity, the phase shift $\phi_{0}$ tends to a constant value. Such a value coincides with the phase shift

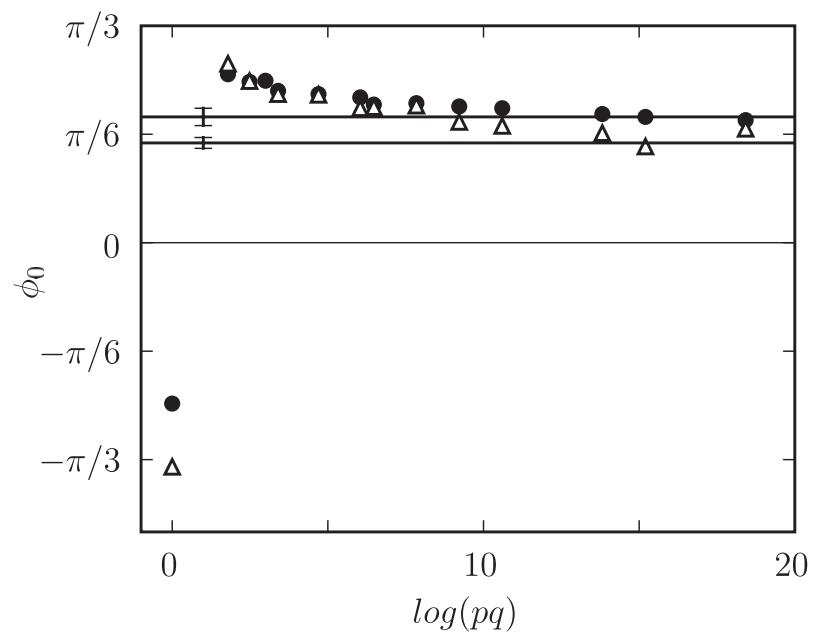

FIG. 2. Phase shift $\phi_{0}$ as a function of the product $p q$ which characterizes the degree of quasiperiodicity of the driving. The two data sets correspond to different amplitudes of the driving: $a=150 \mathrm{kHz}$ for the circles, and $a=75 \mathrm{kHz}$ for the triangles, with $b=c=75 \mathrm{kHz}$ for both data sets. The driving frequencies are $\omega_{1}=2 \pi 100 \mathrm{kHz}, \omega_{2}=(p / q) \omega_{1}$, and the phase of the driving at frequency $\omega_{2}$ is $\delta=\pi / 2$. All other parameters are the same as in Fig. 1. The two horizontal lines indicate the phase shift $\phi_{0}$ for biharmonic drive, i.e., in the absence of the driving at frequency $\omega_{2}$. $\phi_{0}$ measured in the case of pure biharmonic driving (horizontal lines in Fig. 2), which is determined by the finite damping of the atomic motion. We also verified that the asymptotic value of $\phi_{0}$ obtained for large $p q$ is independent of the phase difference $\delta$. These results constitute the experimental proof that in the quasiperiodic limit the only relevant symmetries are those determined by the periodic biharmonic driving and by dissipation. For the specific form of the driving considered, quasiperiodicity therefore restores the symmetries which hold in the absence of the additional driving which produced quasiperiodicity.

We now consider a different driving force, obtained by multiplying the biharmonic driving at frequencies $\omega_{1}, 2 \omega_{1}$ with the driving at frequency $\omega_{2}$. This is done by applying a frequency modulation of beam 1 of the form

$$
\dot{\alpha}(t)=c \sin \left(\omega_{2} t+\delta\right)\left[a \sin \left(\omega_{1} t\right)+b \sin \left(2 \omega_{1} t\right)\right],
$$

which results in a force

$$
\begin{aligned}
F(t)= & -\frac{m c}{k_{z}}\left\{\omega_{2} \cos \left(\omega_{2} t+\delta\right)\left[a \sin \left(\omega_{1} t\right)+b \sin \left(2 \omega_{1} t\right)\right]\right. \\
& \left.+\omega_{1} \sin \left(\omega_{2} t+\delta\right)\left[a \cos \left(\omega_{1} t\right)+2 b \cos \left(2 \omega_{1} t\right)\right]\right\} .
\end{aligned}
$$

We will show that in this case quasiperiodicity results in the total suppression of transport.

We examine first the case of periodic driving. We indicate, as before, $\omega_{2}=(p / q) \omega_{1}$. The period $T$ of $F(t)$ is then $T=q T_{1}=p T_{2}$, with $T_{i}=2 \pi / \omega_{i} \quad(i=1,2)$. Under the transformation $t \rightarrow t+T / 2$ we have $\omega_{1} t \rightarrow$ $\omega_{1} t+q \pi, \omega_{2} t \rightarrow \omega_{2} t+p \pi$. By replacing these transformations in $F(t)$ it is straightforward to see that $F(t)$ satisfies the shift symmetry $F(t)=-F(t+T / 2)$ if $q$ is even and $p$ is odd. In this case directed transport is forbidden. If instead this condition is not satisfied, i.e., if $q$ is odd, directed transport is not forbidden. In this case directed transport is controlled by the $S_{b}$ symmetry, which is realized, in the dissipationless limit, if the driving $F(t)$ is symmetric under time reversal. The symmetry under time reversal depends entirely on the phase $\delta$ of the driving at frequency $\omega_{2}$ : for $q \delta=(n+1 / 2) \pi$, with $n$ integer, the driving is symmetric. Otherwise, the symmetry under time reversal is broken. The current is expected to show a sinusoidal dependence on $q \delta-\pi / 2$, and dissipation will account for an additional shift.

In the experiment, we measured the average atomic velocity as a function of $\delta$ for different values of the driving frequency $\omega_{2}=(p / q) \omega_{1}$, with $p, q$ coprimes. By fitting the data with $v=v_{\max } \sin \left(q \delta+\delta_{0}\right)$, we determined the maximum velocity $v_{\max }$ as a function of $\omega_{2}$. Our results, shown in Fig. 3, demonstrate the relationship between symmetry and transport, valid in the periodic case, discussed above. In fact, a current is observed only for values of the ratio of driving frequencies $\omega_{2} / \omega_{1}=p / q$ with $q$ odd, which is precisely the requirement for the shift symmetry to be broken. 


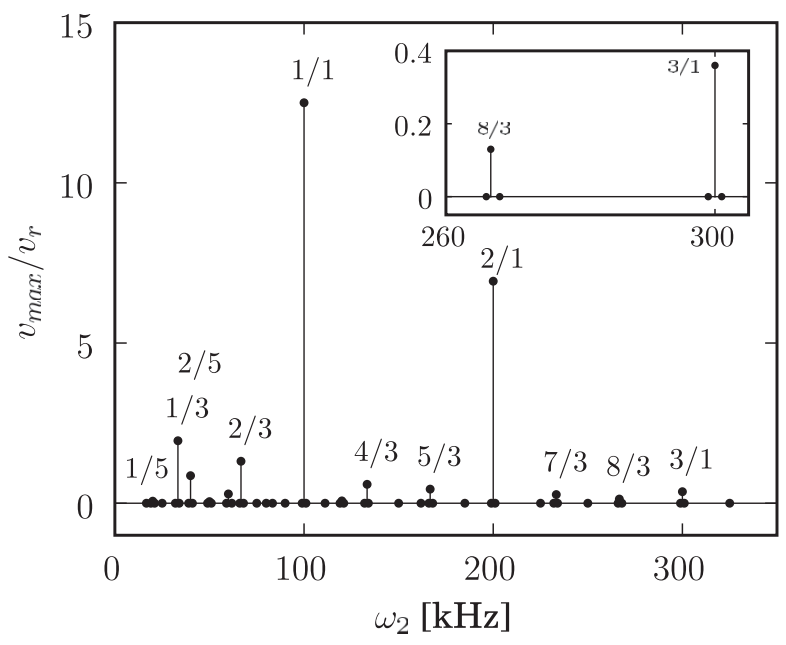

FIG. 3. Maximum average velocity as a function of the driving frequency $\omega_{2}$. The data corresponding to a nonzero velocity are labeled by $p / q=\omega_{2} / \omega_{1}$. The inset magnifies a portion of the plot.

We turn now to the case of quasiperiodic driving. To analyze this case, we introduce the two variables $\psi_{1}=\omega_{1} t$ and $\psi_{2}=\omega_{2} t$, to be treated as independent, and consider the generalized symmetries $\tilde{S}_{a}, \tilde{S}_{b}$. It is immediate to verify that $F$ changes sign under the transformation $\psi_{2} \rightarrow \psi_{2}+$ $\pi$, i.e., $F$ is shift symmetric with respect to $\psi_{2}$. It follows that the system is invariant under the generalized symmetry $\tilde{S}_{a}$. Directed transport is therefore forbidden. In order to study the transition to quasiperiodicity, we rearrange the data of Fig. 3 as a function of $p q$ which characterizes the quasiperiodic character of our driving on the finite duration of the experiment. The results are shown in Fig. 4. It appears that for large $p q$ values the amplitude of the atomic current decreases to zero. This demonstrates that directed transport is destroyed in the quasiperiodic limit, as a result of the restoration of the shift symmetry of the driving.

In conclusion, in this work we studied experimentally the route to quasiperiodicity in a driven ratchet for cold

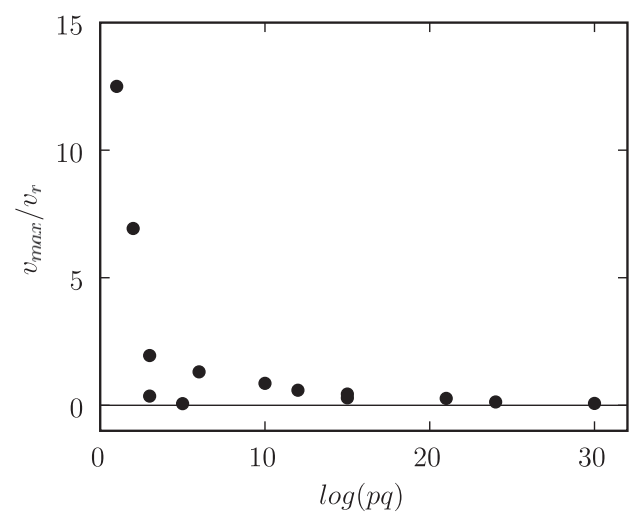

FIG. 4. Maximum average velocity as a function of $p q$, where $p$ and $q$ are the coprimes defined by the ratio of the driving frequencies: $p / q=\omega_{2} / \omega_{1}$. atoms. We examined the relationship between symmetries and transport for two different types of driving. Depending on the specific form of driving, quasiperiodicity results in the complete suppression of transport, or in the restoration of the symmetries which hold for a periodic driving. Our results also demonstrate that by using a multifrequency driving it is possible to precisely control the directed transport by just varying the frequency of one driving. Multifrequency driving also allows one to implement subtle mechanisms of control of the current direction, by introducing appropriate time correlations [2].

We thank S. Flach, H. Maier, W.P. Schleich, and S. Stenholm for useful discussions. Financial support from EPSRC, U.K. and the Royal Society is acknowledged.

[1] A. Ajdari and J. Prost, C.R. Acad. Sci. Paris, Ser. II 315, 1635 (1992); M. O. Magnasco, Phys. Rev. Lett. 71, 1477 (1993); A. Adjari, D. Mukamel, L. Peliti, and J. Prost, J. Phys. I (France) 4, 1551 (1994); R. Bartussek, P. Hänggi, and J.G. Kissner, Europhys. Lett. 28, 459 (1994).

[2] C. R. Doering, W. Horsthemke, and J. Riordan, Phys. Rev. Lett. 72, 2984 (1994).

[3] M. C. Mahato and A. M. Jayannavar, Phys. Lett. A 209, 21 (1995); D. R. Chialvo and M. M. Millonas, Phys. Lett. A 209, 26 (1995).

[4] M.I. Dykman, H. Rabitz, V. N. Smelyanskiy, and B.E. Vugmeister, Phys. Rev. Lett. 79, 1178 (1997); I. Goychuk and P. Hänggi, Europhys. Lett. 43, 503 (1998); D. G. Luchinsky, M. J. Greenall, and P. V.E. McClintock, Phys. Lett. A 273, 316 (2000).

[5] S. Flach, O. Yevtushenko, and Y. Zolotaryuk, Phys. Rev. Lett. 84, 2358 (2000); O. Yevtushenko, S. Flach, Y. Zolotaryuk, and A. A. Ovchinnikov, Europhys. Lett. 54, 141 (2001).

[6] P. Reimann, Phys. Rev. Lett. 86, 4992 (2001).

[7] F. Jülicher, A. Ajdari, and J. Prost, Rev. Mod. Phys. 69, 1269 (1997).

[8] R. D. Astumian and P. Hänggi, Phys. Today 55, No. 11, 33 (2002); P. Reimann, Phys. Rep. 361, 57 (2002).

[9] R. P. Feynman, R. B. Leighton, and M. Sands, The Feynman Lectures on Physics (Addison Wesley, Reading, MA, 1963), Vol. 1, Chap. 46.

[10] H. Linke, T.E. Humphrey, A. Löfgren, A. O. Sushkov, R. Newbury, R. P. Taylor, and P. Omling, Science 286, 2314 (1999).

[11] M. Schiavoni, L. Sanchez-Palencia, F. Renzoni, and G. Grynberg, Phys. Rev. Lett. 90, 094101 (2003).

[12] R. Gommers, S. Bergamini, and F. Renzoni, Phys. Rev. Lett. 95, 073003 (2005).

[13] E. Neumann and A. Pikovsky, Eur. Phys. J. B 26, 219 (2002).

[14] S. Flach and S. Denisov, Acta Phys. Pol. B 35, 1437 (2004).

[15] For a recent review of optical lattices, see G. Grynberg and C. Mennerat-Robilliard, Phys. Rep. 355, 335 (2001). 